\title{
VERIFICATION OF THE CONVENTIONAL MEASURING UNCERTAINTY EVALUATION MODEL WITH MONTE CARLO SIMULATION
}

\author{
Acko, B. \& Godina, A. \\ University of Maribor, Faculty of Mechanical Engineering, Smetanova 17, SI-2000 Maribor, Slovenia \\ E-mail: bojan.acko@uni-mb.si
}

\begin{abstract}
Calibration of gauge blocks by mechanical comparison is widely used for assuring traceability of industrial measurements for the physical quantity "length". In order to diminish uncertainty of calibration, we shall control all influence parameters very precisely. We must be able to predict intervals of changes of these parameters in short and long term intervals and include those changes in the uncertainty budget. Mathematical model of measurement is used for calculating standard uncertainty of the output value (calibration result) from the uncertainties of the input (influence) quantities. However, this calculation is reliable only if the uncertainties of the influence quantities are evaluated accurately. Therefore, we decided to verify the uncertainty calculations from the past by Monte Carlo simulation, which are also proposed by the new draft of the Guide to the Expression Uncertainty in Measurement (GUM). The approach and the results of this verification are presented in the article.
\end{abstract}

Key Words: Measuring Uncertainty Model, Calibration, Gauge Blocks, Monte Carlo Simulation

\section{INTRODUCTION}

The national standard for length in Slovenia is materialized with gauge blocks. These gauge blocks are traceable to the primary standard by calibration in different European national laboratories. The standards from industry and calibration laboratories are calibrated by mechanical comparison with the national standard. The best measurement capability is not comparable with the capabilities of other European national laboratories having interferometric calibration systems but is covering the industrial needs very well. However, our aim is to reduce uncertainty of calibrations without involving interferometric system, which would be too expensive for the industry. Research work in this field is focused in critical uncertainty contributions like calibration of the equipment, as well as environmental and human influences.

As a result of this research the conventional uncertainty evaluation procedure has been supplemented by applying Monte Carlo simulation for evaluating uncertainties of critical influence quantities. Simulation parameters have been chosen according to our extensive experience and knowledge in the field gauge block calibration [1]. Variation intervals for the influence parameters and statistical distributions were defined by studying measurement results of the past ten years.

\section{CONVENTIONAL PROCEDURE FOR UNCERTAINTY EVALUATION}

Conventional procedure for evaluating uncertainty in measurement is described in [2, 3, 4]. This procedure is used worldwide in calibration laboratories, research and national metrology institutes as well as in sophisticated industrial measurements $[5,6,7,8]$. The aim of this 
procedure is to make uncertainty evaluation internationally comparable. As a consequence, parties in trading relations are able to evaluate quality of measuring protocols among each other [9]. Figure 1 comprises only the most important steps to be performed.

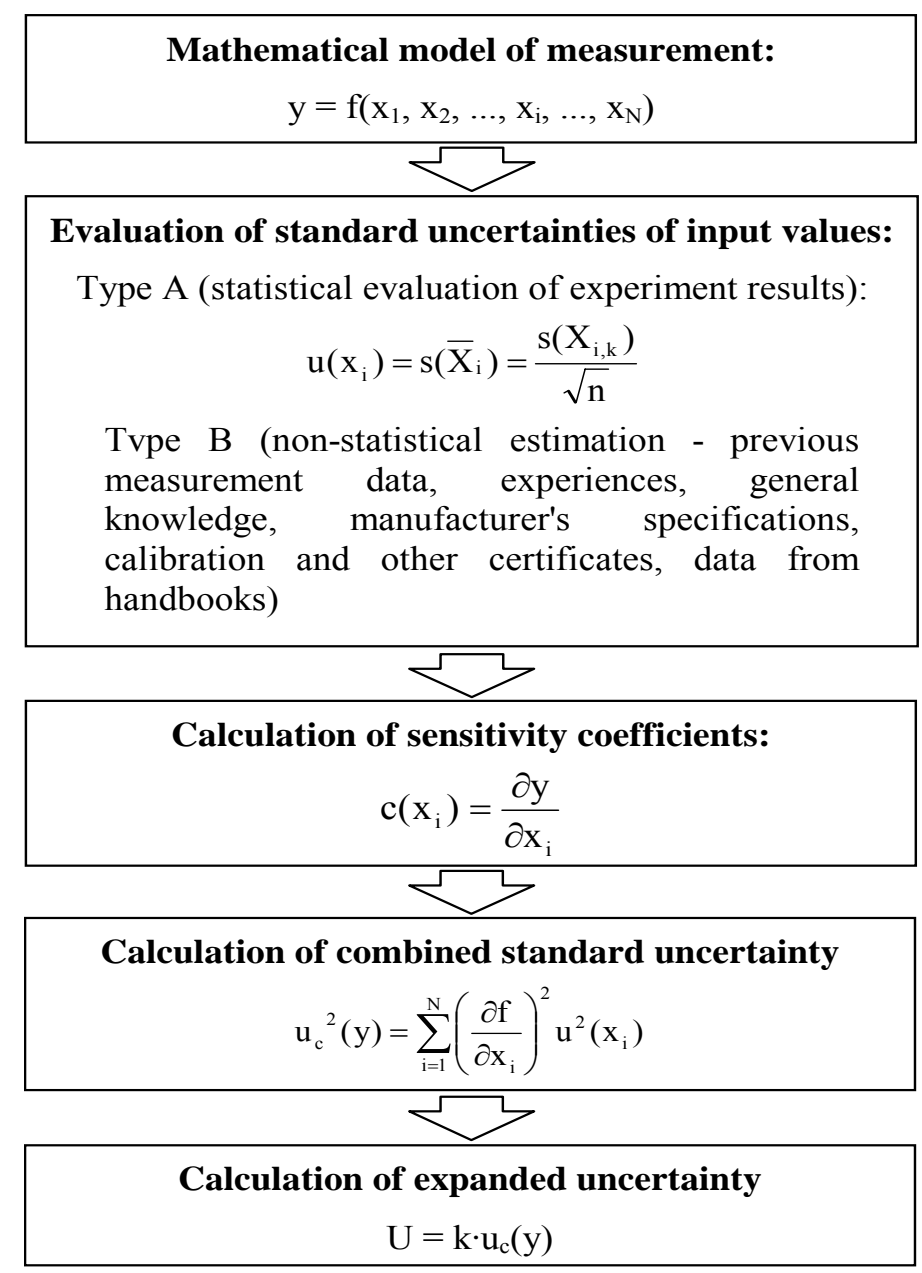

Figure 1: Procedure for evaluating uncertainty of measurement according to [2].

\section{MONTE CARLO SIMULATION IN UNCERTAINTY CALCULATION}

\subsection{Backgrounds and the procedure}

Monte Carlo simulation [10,11] provides a general approach to numerically approximating the distribution function $G(\eta)$ for the value of the output quantity $Y=f(X)$. An estimate of the measurand $Y$, denoted by $y$, is obtained from mathematical model of measurement using input estimates $x_{1}, x_{2}, \ldots, x_{N}$ for the values of the $N$ input quantities $X_{1}, X_{2}, \ldots, X_{N}$. Thus the output estimate $y$, which is the result of the measurement, is given by:

$$
y=f\left(x_{1}, x_{2}, \ldots, x_{N}\right)
$$

That is, $y$ is taken as the arithmetic mean or average of $n$ independent determinations $Y_{k}$ of $Y$, each determination having the same uncertainty and each being based on a complete set of observed values of the $N$ input quantities $X_{i}$ obtained at the same time. This way of averaging may be preferable when $f$ is a nonlinear function of the input quantities $X_{1}, X_{2}, \ldots, X_{N}$, but the two approaches are identical if $f$ is a linear function of the $X_{i}[2]$. 
Although the formula (1) need not provide the most meaningful estimate of the output quantity value, it plays a relevant role within Monte Carlo simulation as an implementation of the propagation of distributions.

Monte Carlo simulation for uncertainty calculations [12] is based on the premise that any value drawn at random from the distribution of possible values of an input quantity is as legitimate as any other such value. Thus, by drawing for each input quantity a value according to its assigned probability density function, the resulting set of values is a legitimate set of values of these quantities. The value of the model corresponding to this set of values constitutes a possible value of the output quantity $Y$. Figure 2 illustrates the propagation of distributions.

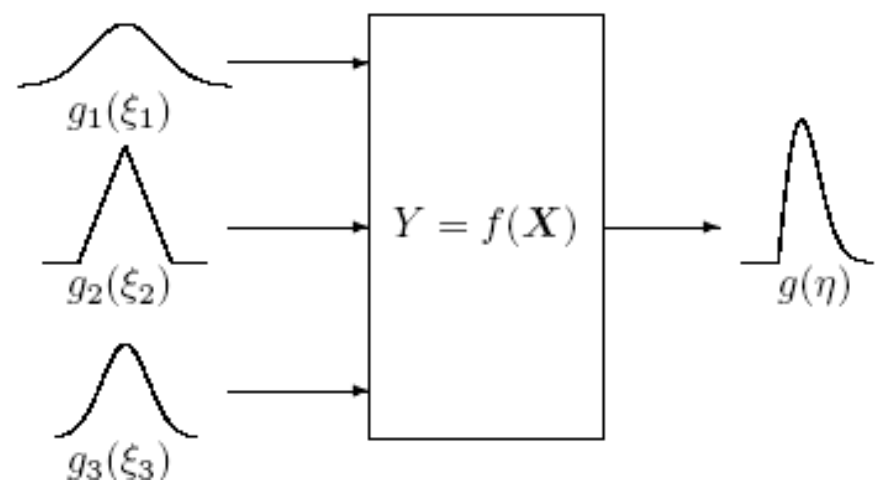

Figure 2: Illustration of the propagation of distributions. The model input quantities are $X=$ $\left(X_{1}, X_{2}, X_{3}\right)^{\mathrm{T}}$. The probability density functions $g_{i}\left(\xi_{i}\right)$, for $X_{i}, i=1,2,3$, are Gaussian, triangular and Gaussian, respectively. The probability density function $g(\eta)$ for the value of the output quantity $Y$ is indicated as being asymmetric, as can arise for nonlinear models.

Consequently, a large set of model values so obtained can be used to provide an approximation to the distribution of possible values for the output quantity. Monte Carlo simulation can be regarded as a generalization of [2] to obtain the distribution for $Y$, rather than the expectation of $Y$.

Monte Carlo simulation operates as follows:

- A sample of size $N$ is generated by independently sampling at random from the probability density function for each $X_{i}, i=1, \ldots, N$. This procedure is repeated a large number of times $(M)$ to yield $M$ independent samples of size $N$ of the set of input quantities. For each such independent sample of size $N$ the resulting model value of $Y$ is calculated;

- These $M$ values of $Y$ are used to provide $\hat{G}(\eta)$, an approximation to the distribution function $G(\eta)$ for the value of $Y$;

- Produce any required statistical quantity from $\hat{G}(\eta)$. Particularly relevant quantities are (a) the expectation of $\hat{G}(\eta)$ as the estimate $y$ of the output quantity value, (b) the standard deviation of $\hat{G}(\eta)$ as the associated standard uncertainty $u(y)$, and (c) two quantiles of $\hat{G}(\eta)$ as the endpoints of a coverage interval $I_{p}(Y)$ for a stipulated coverage probability $p$.

The effectiveness of Monte Carlo simulation to determine a coverage interval for the output quantity value depends on the use of an adequately large value of $M$. Guidance on obtaining such a value and generally on implementing Monte Carlo simulation is available [13]. 
The inputs to the Monte Carlo simulation procedure for the calculation stage are:

- The model $Y=f(X)$ (provided at the formulation stage of uncertainty evaluation).

- The joint probability density function for $X$ (provided at the formulation stage; also see Section 4).

NOTE - This joint probability density function reduces to the collection of the individual probability density functions for $X_{1}, \ldots, X_{N}$ in the case of $N$ mutually independent input quantities.

- The required coverage probability $p$ (e.g., 0.95 , or $95 \%$ ).

- The number $M$ of Monte Carlo trials.

The primary output from the Monte Carlo simulation procedure is a numerical approximation $\hat{G}(\eta)$ to the distribution function $G(\eta)$ for the output quantity value, from which the required quantities can be determined. Monte Carlo simulation as an implementation of the propagation of distributions is shown diagrammatically in Fig. 3 and can conveniently be stated as a step-by-step procedure:

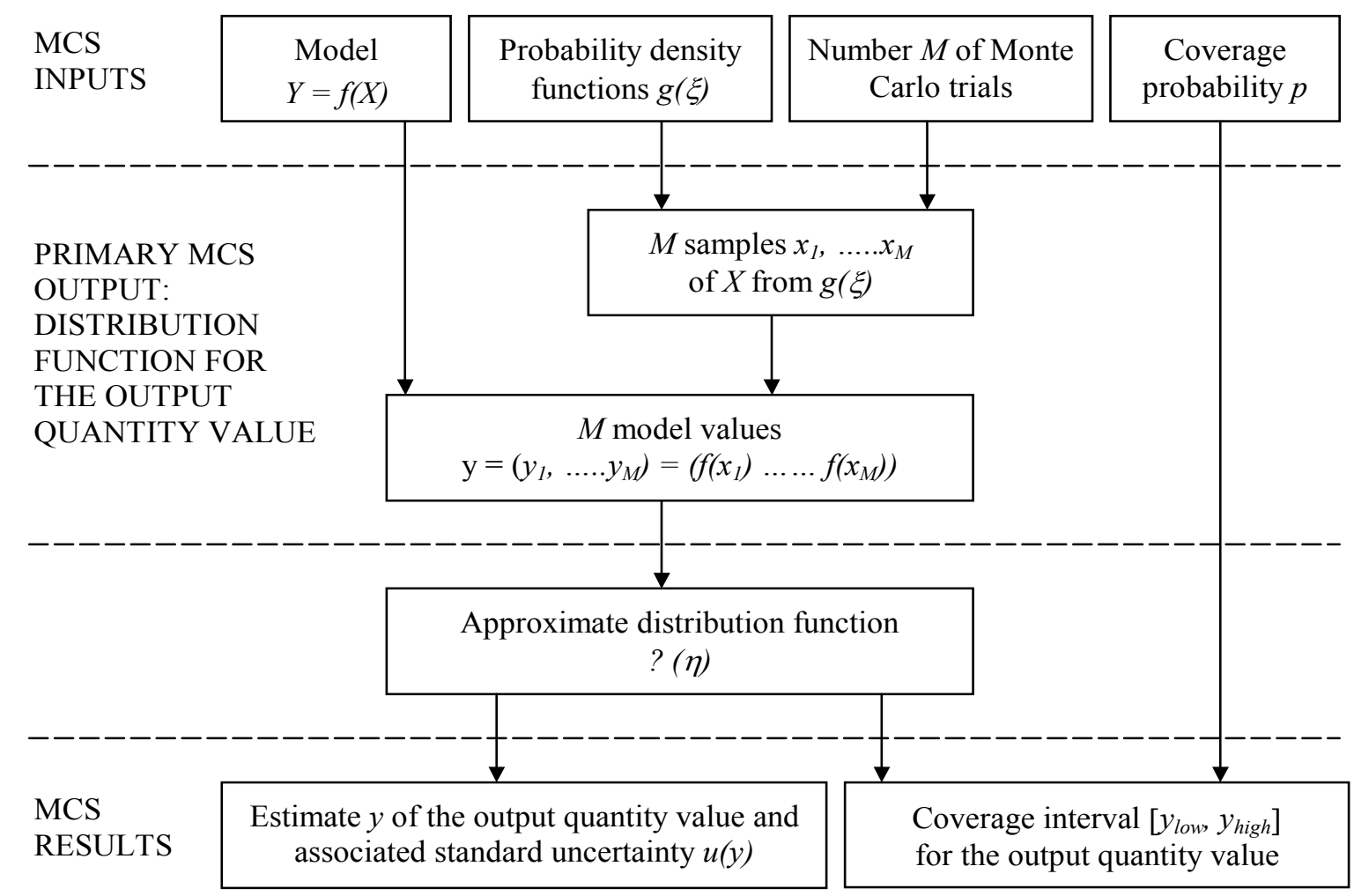

Figure 3: The calculation phase of uncertainty evaluation using Monte Carlo simulation (MCS) to implement the propagation of distributions.

a) The number $M$ of Monte Carlo trials to be made shall be selected;

b) $M$ samples of the (set of $N$ ) input quantities shall be generated;

c) A model giving the corresponding output quantity value shall be evaluated for each sample;

d) The values of the output quantity shall be sorted into non-decreasing order, using the sorted values to approximate the distribution function for the output quantity value;

e) The estimate of the output quantity value and the associated standard uncertainty from the distribution function shall be formed; 
f) The shortest $95 \%$ coverage interval for the output quantity value from this distribution function shall be formed.

\subsection{The number of Monte Carlo trials}

A value of $M$, the number of Monte Carlo trials to be made, needs to be selected. It can be chosen a priori, in which case there will be no direct control over the degree of approximation delivered by the Monte Carlo procedure. The reason is that the number needed to provide a prescribed degree of approximation will depend on the "shape" of the probability density function for the output quantity value and on the coverage probability required. Also, the calculations are stochastic in nature, being based on random sampling. However, a value of $M=10^{6}$ can often be expected to deliver a $95 \%$ coverage interval, having a length with a degree of approximation of one or two significant decimal digits, for the output quantity value.

Because there is no guarantee that this or any specific number will suffice, it is recommended to use a process that selects $M$ adaptively, i.e., as the trials progress. Some guidance in this regard is available [13]. A property of such a process is that it takes a number of trials that is economically consistent with the requirement to achieve the required degree of approximation.

\subsection{Evaluation of the model}

The model is evaluated for each of the $M$ samples from each of the probability density functions for the values of the $N$ input quantities. Specifically, denote the $M$ samples by $x_{l}$, $\ldots, x_{M}$, where the $r^{\text {th }}$ sample $x_{r}$ contains values $x_{1, r}, \ldots, x_{N, r}$, with $x_{i, r}$ a "draw" from the probability density function for $X_{i}$. Then, the model values are:

$$
y_{r}=f\left(x_{r}\right) ; r=1, \ldots \ldots, M
$$

\subsection{Distribution function for the output quantity value}

An approximation $\hat{G}(\eta)$ to the distribution function $G(\eta)$ for the output quantity value $Y$ can be obtained by sorting the values $y_{r}, r=1, \ldots, M$, of the output quantity provided by Monte Carlo simulation into non-decreasing order and denoting the sorted values by $y_{(r)}, r=1, \ldots, M$. Uniformly spaced cumulative probabilities $p r=(r-1 / 2) / M, r=1, \ldots, M$, to the ordered values shall be assigned [12].

$\hat{G}(\eta)$ is the piecewise-linear function joining the $M$ points $\left(y_{(r)}, p_{r}\right), r=1, \ldots, M$ :

$$
\begin{aligned}
& \hat{G}(\eta)=\frac{r-1 / 2}{M}+\frac{\eta-y_{(r)}}{M\left(y_{(r+1)}-y_{(r)}\right)} \\
& y_{(r)} \leq \eta \leq y_{(r+1)}, \quad r=1, \ldots . ., M-1
\end{aligned}
$$

\subsection{The estimate of the output quantity value and the associated standard uncertainty}

The expectation by of the function $\hat{G}(\eta)$ (3) approximates the expectation of the probability density function $g(\eta)$ for the value of $Y$ and is taken as the estimate $y$ of the output quantity value. The standard deviation $u(\hat{y})$ of $\hat{G}(\eta)$ approximates the standard deviation of $g(\eta)$ and is taken as the standard uncertainty $u(y)$ associated with $y . \hat{y}$ can be taken as the arithmetic mean 


$$
\hat{\mathrm{y}}=\frac{1}{\mathrm{M}} \sum_{\mathrm{r}=1}^{\mathrm{M}} \mathrm{y}_{\mathrm{r}}
$$

formed from the $M$ values $y_{l}, \ldots, y_{M}$, and the standard deviation $u(\hat{y})$ determined from:

$$
u^{2}(\hat{y})=\frac{1}{M-1} \sum_{r=1}^{M}\left(y_{r}-\hat{y}\right)^{2}
$$

\section{CONVENTIONAL MEASURING UNCERTAINTY EVALUATION IN MECHANICAL GAUGE BLOCK CALIBRATION}

\subsection{Mathematical model of the measurement}

The length $L$ of the gauge block being calibrated is given by the expression [2]:

where:

$$
L=L e+\Delta L-L e \cdot(\delta \alpha \cdot \theta+\alpha e \cdot \delta \theta)
$$

$L \quad$ - length of the gauge block "B" (gauge being calibrated) at $20{ }^{\circ} \mathrm{C}$

$L_{e} \quad$ - length of the standard gauge "A", given in the calibration certificate at $20{ }^{\circ} \mathrm{C}$

$\Delta L$ - measured difference between the gauge blocks " $\mathrm{A}$ " and " $\mathrm{B}$ "

$\delta \alpha$ - difference of the thermal expansion coefficients of gauges " $\mathrm{A}$ " and " $\mathrm{B}$ "

$\theta$ - deviation of the temperature of the gauge block " $\mathrm{B}$ " from $20{ }^{\circ} \mathrm{C}$

$\alpha e$ - thermal expansion coefficient of the gauge block " $\mathrm{A}$ "

$\delta \theta$ - difference of temperatures of gauge blocks " $\mathrm{A}$ " and " $\mathrm{B}$ "

Measured difference between the gauge blocks " $\mathrm{A}$ " and " $\mathrm{B}$ " is calculated as by the expression:

where:

$$
\Delta L=\Delta L_{a m}+k_{1}+k_{2}
$$

$$
\begin{aligned}
& \Delta L_{a m} \text { - arithmetic mean of the measured difference (experimental) } \\
& k_{1}-\text { comparator's random error } \\
& k_{2} \quad \text { - comparator's systematic error }
\end{aligned}
$$

Deviation of the temperature of the gauge block "B" from $20{ }^{\circ} \mathrm{C}$ consists of the reading from the temperature measurement system, of the difference between the table temperature and the mean temperature of the standard and of temperature variation:

where:

$$
\theta=\theta_{1}+\Delta \theta+\theta_{2}
$$

$\theta_{1} \quad$ - reading from the temperature measurement system

$\Delta \theta$ - difference between the table temperature and the mean temperature of the standard

$\theta_{2} \quad$ - temperature variation 


\subsection{Calculation of combined standard uncertainty}

All evaluated standard uncertainties of the input value estimates for $100 \mathrm{~mm}$ GB are shown in Table I [14].

Table I: Standard uncertainties of the input value estimates for the $100 \mathrm{~mm}$ GB.

\begin{tabular}{|c|c|c|c|c|c|}
\hline $\begin{array}{c}\text { Quantity } \\
X_{i}\end{array}$ & Estimated value & $\begin{array}{l}\text { Standard } \\
\text { uncertainty }\end{array}$ & Distribution & $\begin{array}{l}\text { Sensitivity } \\
\text { coefficient }\end{array}$ & $\begin{array}{l}\text { Uncertainty } \\
\text { contribution }\end{array}$ \\
\hline Le & $100 \mathrm{~mm}$ & $13.3 \mathrm{~nm}$ & normal & 1 & $13.3 \mathrm{~nm}$ \\
\hline$\Delta \mathrm{L}_{\mathrm{am}}$ & $<1 \mathrm{~nm}$ & $1.88 \mathrm{~nm}$ & normal & 1 & $1.88 \mathrm{~nm}$ \\
\hline $\mathrm{k}_{1}$ & $<1 \mathrm{~nm}$ & $15 \mathrm{~nm}$ & normal & 1 & $15 \mathrm{~nm}$ \\
\hline $\mathrm{k}_{2}$ & $<1 \mathrm{~nm}$ & $5 \mathrm{~nm}$ & normal & 1 & $5 \mathrm{~nm}$ \\
\hline$\delta \alpha$ & $0^{\circ} \mathrm{C}^{-1}$ & $0,58 \cdot 10^{-6}{ }^{\circ} \mathrm{C}^{-1}$ & rectangular & $-\mathrm{L}_{\mathrm{e}} \cdot \theta$ & $5.8 \mathrm{~nm}$ \\
\hline$\theta_{1}$ & $0^{\circ} \mathrm{C}$ & $2,5 \cdot 10^{-3}{ }^{\circ} \mathrm{C}$ & normal & $-\mathrm{L}_{e} \cdot \delta \alpha$ & $0.25 \mathrm{~nm}$ \\
\hline$\Delta \theta$ & $0^{\circ} \mathrm{C}$ & $0.033^{\circ} \mathrm{C}$ & normal & $-\mathrm{L}_{\mathrm{e}} \cdot \delta \alpha$ & $3.3 \mathrm{~nm}$ \\
\hline$\theta_{2}$ & $0^{\circ} \mathrm{C}$ & $0.035^{\circ} \mathrm{C}$ & rectangular & $-\mathrm{L}_{\mathrm{e}} \cdot \delta \alpha$ & $3.5 \mathrm{~nm}$ \\
\hline$\alpha e$ & $11.6 \cdot 10^{-6}{ }^{\circ} \mathrm{C}^{-1}$ & $0.29 \cdot 10^{-60} \mathrm{C}^{-1}$ & rectangular & $-\mathrm{L}_{\mathrm{e}} \delta \theta$ & $2.9 \mathrm{~nm}$ \\
\hline$\delta \theta$ & $0^{\circ} \mathrm{C}$ & $0.0112^{\circ} \mathrm{C}$ & rectangular & $-\mathrm{L}_{\mathrm{e}} \cdot \alpha_{\mathrm{e}}$ & $12.32 \mathrm{~nm}$ \\
\hline & & & & Total: & $25.45 \mathrm{~nm}$ \\
\hline
\end{tabular}

In our case, combined standard uncertainty (after rounding it up) for the $100 \mathrm{~mm} \mathrm{~GB}$ under the best possible conditions is $u=26 \mathrm{~nm}$.

\subsection{Expanded uncertainty}

When using factor $k=2$, absolute expanded uncertainty of calibration for the $100 \mathrm{~mm}$ GB is:

$$
U=52 \mathrm{~nm} \text {. }
$$

\section{VERIFICATION OF THE CONVENTIONAL UNCERTAINTY EVALUATION BY USING MONTE CARLO SIMULATION}

For the Monte Carlo simulation of the uncertainty evaluation in mechanical gauge block calibration the same mathematical model of the measurement (6) as in conventional procedure of uncertainty evaluation, together with assigned probability density functions (Table II), was used. The number of Monte Carlo trials was set to $M=100,000$ [15].

Figure 4 shows the Monte Carlo simulation's resulting histogram with corresponding normal fit and the shortest $95 \%$ coverage interval. 
Table II: Input values and probability density functions for the $100 \mathrm{~mm}$ GB.

\begin{tabular}{|c|lc|}
\hline $\begin{array}{c}\text { Quantity } \\
\boldsymbol{X}_{\boldsymbol{i}}\end{array}$ & \multicolumn{1}{|c|}{ Probability density function $\boldsymbol{g}\left(\boldsymbol{x}_{\boldsymbol{i}}\right)$} \\
\hline $\mathrm{Le}$ & normal distribution $\quad(\mathrm{M} ; 100 \mathrm{~mm} ; 13.3 \mathrm{~nm})$ \\
\hline$\Delta \mathrm{L}_{\mathrm{am}}$ & normal distribution $\quad(\mathrm{M} ; 0 \mathrm{~mm} ; 1.88 \mathrm{~nm})$ \\
\hline $\mathrm{k}_{1}$ & normal distribution $\quad(\mathrm{M} ; 0 \mathrm{~mm} ; 15 \mathrm{~nm})$ \\
\hline $\mathrm{k}_{2}$ & normal distribution $\quad(\mathrm{M} ; 0 \mathrm{~mm} ; 5 \mathrm{~nm})$ \\
\hline$\delta \alpha$ & rectangular distribution $\left(\mathrm{M} ;-1 \cdot 10^{-6}{ }^{\circ} \mathrm{C}^{-1} ; 1 \cdot 10^{-6}{ }^{\circ} \mathrm{C}^{-1}\right)$ \\
\hline$\theta_{1}$ & normal distribution $\quad\left(\mathrm{M} ; 0^{\circ} \mathrm{C} ; 2,5 \cdot 10^{-3}{ }^{\circ} \mathrm{C}\right)$ \\
\hline$\Delta \theta$ & normal distribution $\quad\left(\mathrm{M} ; 0{ }^{\circ} \mathrm{C} ; 0.033^{\circ} \mathrm{C}\right)$ \\
\hline$\theta_{2}$ & rectangular distribution $\left(\mathrm{M} ;-0.03{ }^{\circ} \mathrm{C} ; 0.03{ }^{\circ} \mathrm{C}\right)$ \\
\hline$\alpha \mathrm{e}$ & rectangular distribution $\left(\mathrm{M} ; 11.1 \cdot 10^{-6}{ }^{\circ} \mathrm{C}^{-1} ; 12.1 \cdot 10^{-6}{ }^{\circ} \mathrm{C}^{-1}\right)$ \\
\hline$\delta \theta$ & rectangular distribution $\left(\mathrm{M} ;-0.02^{\circ} \mathrm{C} ; 0.02{ }^{\circ} \mathrm{C}\right)$ \\
\hline
\end{tabular}

$$
\begin{array}{llrl}
\mathrm{Y}_{0025} & =99.99996 \mathrm{~mm} & \operatorname{mean}(\mathrm{L}) & =100.00000 \mathrm{~mm} \\
\mathrm{Y}_{0975} & =100.00004 \mathrm{~mm} & & \operatorname{stdev}(\mathrm{L})=21.0 \mathrm{~nm}
\end{array}
$$

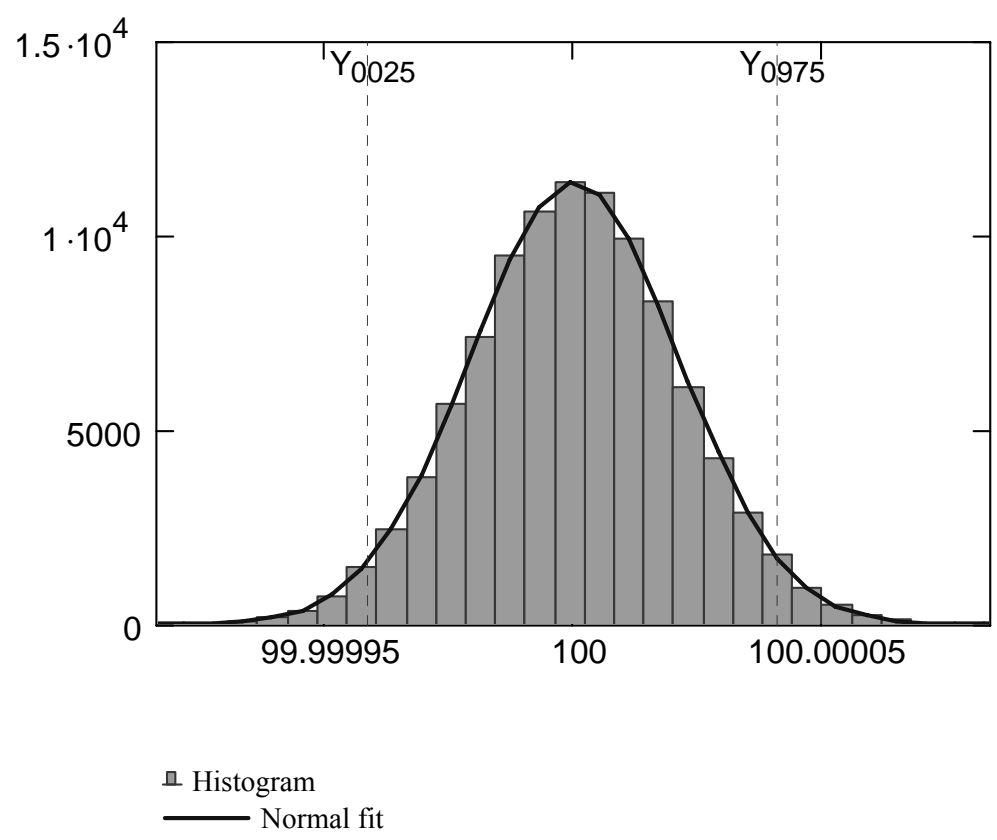

Figure 4: Probability density function $g(L)$ for mechanical calibration of the $100 \mathrm{~mm}$ GB.

\section{CONCLUSIONS}

Mathematical model of measurement was used for calculating standard uncertainty of the gauge block calibration by mechanical comparison. By Monte Carlo simulation we wanted to verify the correctness and completeness of uncertainties of the influence quantities, together with their accurate evaluation. 
Comparison of uncertainty, calculated by conventional measuring uncertainty evaluation, with the result of Monte Carlo simulation, shows small difference of only 4,5 nm. Agreement in results in such an extent verifies our mathematical model of mechanical gauge block calibration, evaluation of its standard uncertainties and performed calculations.

Intensions for our future work are implementation of Monte Carlo simulation for verification of more complex mathematical models like models of form calibration.

\section{REFERENCES}

[1] Acko, B.; Sostar, A. (2002). Modification of the model for measurement uncertainty evaluation in a gauge-block calibration based on measurement automation, Strojniski vestnik, Vol. 48, No. $1,9-16$

[2] International Standardization Organization (1995). ISO Guide to the Expression of Uncertainty in Measurement

[3] Acko, B. (2003). A universal model for evaluating measuring uncertainty in calibration, International Journal of Simulation Modelling, Vol. 2, No. 4, 121-129

[4] Doiron, T.; Beers, J. (2005). The Gauge Block Handbook, NIST Monograph 180, http://emtoolbox.nist.gov/Publications/NISTMonograph180.asp, accessed on 3.5.2005

[5] Thalmann, R.; Baechler, H. (2003). Issues and advantages of gauge block calibration by mechanical comparison, Proceedings of SPIE, Vol. 5190, 62-69

[6] Taylor, B. N.; Kuyatt, C. E. (1993). Guidelines for Evaluating and Expressing the Uncertainty of NIST Measurement Results, NIST Technical Note 1297

[7] Faust, B.; Stoup, J.; Stanfield, E. (1998). Minimizing error sources in gage block mechanical comparison measurements, Proceedings of SPIE, Vol. 3477, 127-136

[8] Kacker, R.; Jones, A. (2003). On use of Bayesian statistics to make the Guide to the Expression of Uncertainty in Measurement consistent, Metrologia, Vol. 40, No. 5, 235-248

[9] Dietrich, C. F. (1991). Uncertainty, Calibration and Probability, Adam Hilger, Bristol

[10] Fisher, G. S. (1996). Monte Carlo. Springer-Verlag, New York

[11] Robert, C. P., Casella, G. (1999). Monte Carlo Statistical Methods, Springer-Verlag, New York

[12] Guide to the Expression of Uncertainty in Measurement, Supplement 1: Numerical Methods for the Propagation of Distributions (Draft), http://www.ncsli.org/committees/133/index.cfm, accessed on 3.5.2005

[13] Cox, M. et al (2001). Best Practice Guide No. 6. Uncertainty and statistical modeling, Technical report, National Physical Laboratory, Teddington

[14] Godina, A.; Acko, B. (2004). Influence of the Measurement Force on the Uncertainty of the Gauge Block Comparator, VDI-Berichte 1860 (Duesseldorf: VDI)

[15] Druzovec, M. et al (2001). Diagnostics and a qualitative model, International journal of medical informatics, No. 63, 51-60 